\title{
EVOLUÇÃO CLÍNICA E ENDOSCÓPICA APÓS FUNDOPLICATURA PARA TRATAMENTO DA DOENÇA DO REFLUXO GASTROESOFÁGICO
}

\author{
Alessandra Maria Borges VICENTE', Sílvia Regina CARDOSO², \\ Maria de Fátima Correia Pimenta SERVIDONI², Luciana Rodrigues de MEIRELLES ${ }^{3}$, \\ Joaquim Murray Bustorff SILVA ${ }^{4}$ e Elizete Aparecida Lomazi da COSTA-PINTO5
}

\begin{abstract}
RESUMO - Contexto - O tratamento cirúrgico da doença do refluxo gastroesofágico está indicado para pacientes com doença crônica, associada ou não a complicações. A fundoplicatura é frequentemente indicada em substituição ao uso contínuo dos inibidores de bomba de prótons, para pacientes sem resposta, resposta parcial, ou dependentes de tratamento medicamentoso, ou ainda, quando houver recurrência dos sintomas com a descontinuação das medicações. No período pós-operatório, ocorrências de desmanche da válvula com recurrência da doença do refluxo indicam a necessidade de monitorização do procedimento cirúrgico. A avaliação do funcionamento da válvula, baseada na presença de sintomas, tem se mostrado instrumento ineficaz para essa monitorização. Objetivo - Identificar a frequência de anormalidades na válvula antirrefluxo e a frequência de complicações pépticas do esôfago no período de pós-operatório tardio da fundoplicatura em crianças e adolescentes. Métodos - Em estudo transversal, prospectivo e descritivo, em que foram avaliados 45 pacientes (idade $=16$ meses a 16,9 anos) que tinham realizado fundoplicatura de Nissen num período de 12 a 30 meses prévios à avaliação. $\mathrm{O}$ aspecto da fundoplicatura e da mucosa esofágica foram avaliados por meio de endoscopia digestiva alta e estudo histológico. Resultados - Doentes com encefalopatia crônica corresponderam a 26/45 (57,8\%) dos casos. Válvula antirrefluxo intacta foi identificada em 41/45 $(91,1 \%)$ dos pacientes. A fundoplicatura foi efetiva no tratamento do processo inflamatório esofágico, mesmo quando identificadas subestenose ou estenose de esôfago, no préoperatório. As complicações identificadas foram esofagite péptica (6/45 pacientes) e necessidade de nova fundoplicatura (2/45 pacientes). A presença de anormalidades na válvula antirrefluxo associou-se ao achado de esofagite péptica $(P=0,005)$. Dois pacientes receberam o diagnóstico de esôfago de Barrett. Conclusão - A fundoplicatura pela técnica de Nissen se mostrou eficiente no controle das complicações esofágicas da doença do refluxo gastroesofágico. A endoscopia digestiva alta foi um exame útil na monitorização pós-operatória de crianças que realizaram fundoplicatura por doença do refluxo gastroesofágico. Permitiu avaliar a condição da válvula antirrefluxo e diagnosticar complicações relacionadas à recidiva da doença, mesmo em pacientes assintomáticos.
\end{abstract}

DESCRITORES - Fundoplicatura. Refluxo gastroesofágico, cirurgia. Esofagite. Estenose esofágica. Esôfago de Barrett. Endoscopia gastrointestinal. Criança.

\section{INTRODUÇÃO}

Na doença do refluxo gastroesofágico (DRGE), a passagem involuntária do conteúdo gástrico para o esôfago causa repercussão negativa sobre a saúde da criança( ${ }^{(2)}$. Podem ocorrer alterações relacionadas à nutrição, à mucosa esofágica, à função respiratória ou ao aparecimento de sintomas neurocomportamentais ${ }^{(6)}$. Para as crianças com DRGE associada a complicações graves e/ou à doença crônica, as melhores opções terapêuticas são o tratamento medicamentoso com inibidor de bomba de prótons e a cirurgia antirrefluxo ${ }^{(14,35)}$.

As recomendações para o uso dos inibidores de bomba de prótons em crianças e adolescentes são variadas nos diversos países. A falta de dados sobre o uso desses fármacos em pacientes pediátricos, em longo prazo, é lacuna que limita o uso clínico. O tempo máximo de uso dessas medicações, registrado como seguro e eficaz, foi de 36 meses $^{(14,35)}$.

A técnica de fundoplicatura descrita por Nissen é a mais frequentemente usada na faixa etária pediátrica ${ }^{(6,15,38)}$. O objetivo da cirurgia é interromper o refluxo gastroesofágico por meio de uma combinação de mecanismos antirrefluxo. $\mathrm{O}$ tratamento cirúrgico está indicado, principalmente, quando há falha do tratamento clínico ou na presença da DRGE complicada. Fundoplicatura é mais frequentemente indicada em crianças maiores e naquelas com risco para DRGE grave, entre elas, encefalopatas crônicos, em particular

Trabalho realizado na Disciplina de Gastroenterologia, Departamento de Pediatria, Universidade Estadual de Campinas (UNICAMP)

1 Faculdade de Ciências Médicas, Universidade Estadual de Campinas - FCM-UNICAMP; ${ }^{2}$ Serviço de Endoscopia Pediátrica, Hospital das Clínicas - HC-UNICAMP. Departamentos de ${ }^{3}$ Anatomia Patológica, Gastrocentro-UNICAMP; ${ }^{4}$ Cirurgia Pediátrica e Pediatria - FCM-UNICAMP.

Correspondência: Dra. Alessandra Maria Borges Vicente - Rua Eunice Virginia Ramos Navero, 310, casa 14 - 13087-765 - Campinas, SP. E-mail: ambv@bol.com.br 
a encefalopatia crônica não progressiva (ECNP), pacientes com doença respiratória crônica ou pacientes operados por atresia de esôfago. Nesses pacientes, morbidade pós-operatória e insucesso cirúrgico são mais comuns ${ }^{(6,26)}$.

$\mathrm{O}$ índice de falha da técnica cirúrgica pode variar de $5 \%$ a $20 \%$ das crianças operadas, dependendo principalmente, da presença de doenças associadas, sendo que as maiores taxas de recurrência ocorrem em pacientes encefalopatas crônicos ou com problemas respiratórios crônicos ${ }^{(3,28,37,38)}$. Do ponto de vista anatômico, as principais causas de recurrência da DRGE são desmanche parcial ou total da fundoplicatura ${ }^{(25)} \mathrm{e}$, menos frequentemente, a migração da válvula antirrefluxo para o tórax. A válvula cirúrgica pode gradualmente tornar-se incompetente, mas não existem informações precisas sobre latência e regularidade dessa ocorrência ${ }^{(26)}$. O tempo ideal de seguimento nos pacientes operados e o instrumento adequado de monitorização não estão adequadamente estabelecidos.

O presente estudo foi realizado com o objetivo de identificar, em crianças e adolescentes que realizaram fundoplicatura para tratamento de DRGE, a frequência de anormalidades na válvula antirrefluxo e de complicações relacionadas à DRGE. A avaliação foi feita por meio do exame de endoscopia digestiva alta (EDA), comparando as frequências entre os pacientes, de acordo com a presença de encefalopatia crônica. As informações provenientes poderão ser úteis para a definição de um protocolo de acompanhamento pós-operatório.

\section{MÉTODOS}

\section{Casuística}

Estudo transversal e descritivo foi conduzido no período de maio de 2004 a fevereiro de 2007. As características pósoperatórias da válvula antirrefluxo, realizada para tratamento de DRGE em 45 crianças e adolescentes foram examinadas por meio de EDA. Esses pacientes foram identificados pelos registros cirúrgicos num hospital escola e convocados por telefone e/ou telegrama.

Os critérios de inclusão no estudo foram: 1. pós-operatório de um único procedimento cirúrgico de fundoplicatura para DRGE, e 2. diagnóstico de DRGE firmado com bases clínicas e investigação mínima com um exame complementar.

Foram excluídos os doentes com malformação esofágica ou estenose cáustica de esôfago, os com dados incompletos no prontuário, os que não concordaram em participar do estudo e aqueles não encontrados pelas vias de comunicação do Serviço Social do hospital, por meio de telefone ou telegrama.

Noventa e três pacientes foram operados durante o período considerado. Os motivos que não permitiram a inclusão de 48 pacientes no estudo foram: não localização $(n=22)$, não concordância para realizar a avaliação endoscópica $(n=8)$, óbito $(n=7)$, atresia de esôfago ou estenose cáustica de esôfago associada à DRGE $(n=5)$, mais que um procedimento cirúrgico de fundoplicatura $(n=3)$, cirurgia realizada sem confirmação por exame complementar da DRGE $(\mathrm{n}=2)$ e paciente operado com idade próxima a 19 anos que saiu do acompanhamento no serviço $(n=1)$.

A indicação da cirurgia foi baseada na história clínica e nos exames complementares de investigação. A maior parte dos pacientes $(62,2 \%)$ fez dois ou mais exames complementares diagnósticos, confirmando DRGE.

Todos os pacientes haviam sido tratados com medidas posturais antirrefluxo, agentes procinéticos, inibidores do receptor $\mathrm{H}_{2}$ da histamina e/ou inibidores de bomba de prótons, previamente à indicação cirúrgica.

Em $35(77,8 \%)$ pacientes a cirurgia foi a descrita por Nissen, com via de acesso laparoscópica e nos outros 10 por laparotomia (22,2\%). Em 17 pacientes a fundoplicatura foi associada à gastrostomia, pela técnica de Stamm, em 3 pacientes à piloroplastia, pela técnica de Heineke-Mikulicz, e em 3 pacientes a fundoplicatura foi combinada à gastrostomia e piloroplastia.

\section{Métodos}

De maio de 2004 a fevereiro de 2007, os pacientes incluídos foram convocados para entrevista e exame de EDA. Nos meses de janeiro e fevereiro de 2007, os prontuários foram revisados e registrados dados referentes à evolução (necessidade de nova fundoplicatura e óbito). Desta maneira, o tempo total de avaliação foi de 12 a 48,2 meses.

Os exames de EDA foram realizados pela equipe de Endoscopia Pediátrica do hospital escola, utilizando-se o aparelho de videoendoscopia da marca Pentax ou Olympus. Dedicou-se atenção especial para a região do cárdia, por meio da manobra de retroversão, para visualização da válvula antirrefluxo.

Foram utilizados como critérios diagnósticos de anormalidades da fundoplicatura, os descritos por LUOSTARINEN ${ }^{(23)}$ :

- migração da válvula antirrefluxo para o tórax (válvula íntegra, porém deslocada), hiato diafragmático alargado, ou

- fundoplicatura desfeita parcial ou totalmente: presença de pregas frouxas e distorcidas (parcial), ou ausência de pregas (total), ou

- migração da válvula antirrefluxo para o tórax associado à fundoplicatura desfeita parcial ou totalmente.

A classificação utilizada para diagnóstico endoscópico de esofagite foi a do Sistema Los Angeles, criado em 1994 e modificado em $1999^{(21,22,27,30)}$.

Foi realizada biopsia em esôfago distal em 37 dos 45 pacientes (82,2\%). Obteve-se pelo menos dois fragmentos, com distância proximal mínima de $2 \mathrm{~cm}$ da transição esôfago-gástrica. $\mathrm{O}$ estudo histológico foi realizado sempre pelo mesmo profissional e pelo pesquisador. Os dois fragmentos de tecido foram mensurados e avaliados, sendo considerado o de maior dimensão, para graduação histológica. Não houve acesso ao laudo histológico prévio e ao laudo endoscópico do paciente durante a revisão de lâminas. A classificação utilizada para diagnóstico histológico de esofagite foi a de GEBOES, publicada em $1991^{(9,34)}$ :

- grau 0: normal

- grau 1: a) hiperplasia de zona basal

b) alongamento de papila estromal

c) congestão vascular

- grau 2: células polimorfonucleares no epitélio, lâmina própria ou ambos

- grau 3: polimorfonucleares com defeito epitelial

- grau 4: ulceração

- grau 5: epitélio colunar aberrante 


\section{Análise dos dados}

Os dados obtidos foram armazenados e analisados com o programa SPSS versão 7.5 (SPSS Inc. Chicago, IL, EUA). A comparação de frequências para variáveis categóricas, entre dois grupos, encefalopatas ou não, foi feita por meio de tabelas 2 X 2 e aplicado teste do qui ao quadrado ou teste exato de Fisher. $\mathrm{O}$ nível de significância adotado foi de $5 \%$.

Este estudo foi aprovado pelo Comitê de Ética em Pesquisa da Faculdade de Ciências Médicas da Universidade Estadual de Campinas, SP, sem restrições (parecer número 160/2004). Todos os responsáveis pelos pacientes assinaram termo de consentimento informado.

\section{RESULTADOS}

\section{Descritivo da casuística}

O estudo avaliou 45 pacientes que realizaram fundoplicatura para tratamento de DRGE. Vinte e seis pacientes $(57,8 \%)$ eram do sexo masculino. A idade, no momento do estudo e respectiva avaliação endoscópica pós-operatória, variou de 16 meses a 16,9 anos, com média de 7,4 anos, mediana de 7,6 anos e desvio padrão de 4,4 anos, coincidindo com período pós-operatório mínimo de 12 e máximo de 30 meses. Oito pacientes foram operados com idade inferior a 1 ano e 26 tinham encefalopatia crônica $(57,8 \%)$.

\section{EDA pré-operatória}

Vinte e seis pacientes tinham realizado EDA no pré-operatório, os diagnósticos foram de esofagite péptica (22/26), subestenose ou estenose de esôfago (7/26), hérnia hiatal por deslizamento (7/26) e suspeita de esôfago de Barrett (1/26), não confirmada na biopsia.

\section{Complicações imediatas pós-fundoplicatura}

Sete pacientes $(15,5 \%)$ apresentaram complicações no pósoperatório imediato ou precoce, a saber, pneumonia, pneumotórax, infecção no sítio de gastrostomia, prolapso de mucosa gástrica pelo orifício da gastrostomia com sangramento, e deiscência da ferida operatória. Somente esta última complicação foi tratada com novo procedimento cirúrgico. Quase metade (42,8\%) dessas complicações ocorreu em pacientes menores de 1 ano e operados via laparotomia. Embora a frequência de complicações entre as crianças com encefalopatia crônica $(71,5 \%)$ tenha sido superior à observada entre as crianças neurologicamente saudáveis $(28,5 \%)$, essa diferença não foi significante. A frequência de complicações foi superior quando a cirurgia foi realizada por laparotomia $(4 / 10)$ versus a via videolaparoscópica (3/35), $P=0,034$ (teste exato de Fisher).

\section{EDA pós-operatória}

Quanto aos achados endoscópicos pós-operatórios, verificou-se retorno ou persistência de esofagite péptica em 6 dos 45 pacientes (13,3\%). Além deste achado, foram observados cinco casos de hérnia paraesofagiana, dos quais quatro eram encefalopatas crônicos. Em três dessas crianças a hérnia estava associada à presença de esofagite erosiva.
Em três pacientes a válvula antirrefluxo apresentava deslocamento para o tórax, um dos quais evoluiu para o desmanche parcial da válvula. Este último paciente foi posteriormente reoperado para a correção deste problema. Um paciente apresentava desmanche total da válvula, associado à subestenose de esôfago e esofagite péptica grau D de Los Angeles, sendo necessária nova fundoplicatura.

Não houve diferença significante em relação aos achados endoscópicos relacionados ao esôfago entre os pacientes com ou sem encefalopatia crônica (Tabela 1).

TABELA 1. Achados endoscópicos pós-operatórios de fundoplicatura à Nissen, em 45 pacientes, analisados de acordo com a presença de encefalopatia crônica*

\begin{tabular}{lcc}
\hline EDA & \multicolumn{2}{c}{ EC** $^{*}$} \\
\cline { 2 - 3 } Pós-operatória & Sim & Não \\
& $\mathbf{n}(\%)$ & $\mathbf{n}(\%)$ \\
\hline Normal & $19(73)$ & $17(89)$ \\
Esofagite A** & $3(11)$ & $2(11)$ \\
Esofagite $\mathrm{D}^{\infty}+$ subestenose & $1(3,8)$ & - \\
Subestenose & $1(3,8)$ & - \\
Suspeita de Barrett & $2(7,6)$ & - \\
Total & $26(100)$ & $19(100)$ \\
\hline
\end{tabular}

* encefalopatia crônica, $\cdot P=0,26$, teste exato de Fischer (encefalopatia versus anormalidade endoscópica) ** esofagite grau A de Los Angeles

\#01 paciente subestenose + suspeita de esôfago de Barrett

Ocorreu melhora da inflamação no esôfago após a cirurgia na maioria dos pacientes com anormalidades endoscópicas no pré-operatório. A esofagite desapareceu ou tornou-se mais leve (grau A ou B de Los Angeles) em todos os exames de EDA no pós-operatório, exceto em um paciente, com diagnóstico de esofagite grau D de Los Angeles, no pré e pós-operatório.

O status pós-operatório foi classificado como fundoplicatura continente e bem posicionada em 41 pacientes, fundoplicatura intratorácica em 3 e desmanche total da fundoplicatura em 1 caso. Não houve associação entre ocorrência de encefalopatia e alteração endoscópica da fundoplicatura, $P=0,62$, teste de Fisher (Tabela 2).

TABELA 2. Distribuição dos pacientes com e sem encefalopatia crônica em relação aos achados endoscópicos relacionados à fundoplicatura $(P=0,67$; teste exato de Fisher)

\begin{tabular}{|c|c|c|}
\hline \multirow[t]{2}{*}{ Condição da fundoplicatura } & \multicolumn{2}{|c|}{ Encefalopatia crônica } \\
\hline & $\begin{array}{c}\text { Sim } \\
\mathrm{n}(\%)\end{array}$ & $\begin{array}{c}\text { Não } \\
\text { n (\%) }\end{array}$ \\
\hline Normal & $23(88,5)$ & $18(95)$ \\
\hline Intratorácica/desmanche total & $3(11,5)$ & $1(5)$ \\
\hline Total & $26(100)$ & $19(100)$ \\
\hline
\end{tabular}

A presença de esofagite péptica esteve relacionada à presença de anormalidades na válvula antirrefluxo, $P=0,005$, teste exato de Fisher.

A condição da fundoplicatura não se correlacionou com a presença de esofagite no exame endoscópico de pré-operatório 
$(P=0,474$, teste exato de Fisher), assim como, não houve correlação com a presença de esofagite grave, considerando graus $\mathrm{C}$ e D de Los Angeles ( $P=0,087$, teste exato de Fisher). Não foi verificada correlação estatística entre sucesso da fundoplicatura e realização de gastrostomia $(P=0,368$, teste exato de Fisher) e piloroplastia $(P=0,713$, teste exato de Fisher) no mesmo ato cirúrgico.

A histologia do esôfago distal foi analisada em 36 dos 45 pacientes; em 1 paciente a amostra foi insuficiente e em outros 8 a amostra não foi colhida, já que no serviço rotineiro, a biopsia só era realizada quando havia anormalidades da mucosa esofágica. A partir do oitavo paciente incluído no estudo, os autores passaram a colher amostra para exame histológico em todos os casos. Um diagnóstico de esôfago de Barrett foi confirmado de imediato ao exame histológico em um paciente com subestenose associada e esofagite grau 2. Este paciente apresentava estenose de esôfago ao exame endoscópico de pré-operatório e necessitou de dilatação. $\mathrm{O}$ outro paciente com suspeita endoscópica de esôfago de Barrett, desde o exame de pré-operatório e com subestenose de esôfago associada, apresentava biopsia com esofagite grau 1, resolução da subestenose e ausência de confirmação inicial do diagnóstico de esôfago de Barrett. Essa confirmação histológica ocorreu após a segunda EDA de controle no pós-operatório (8 meses após a primeira endoscopia de seguimento).

\section{Evolução dos sintomas no pós-operatório}

A maior parte dos pacientes $(82,2 \%)$ apresentava queixas digestivas no pré-operatório. A metade $(53,3 \%)$ relatou melhora ou desaparecimento dos sintomas no pós-operatório e 20 pacientes $(44,5 \%)$ relataram melhora parcial com persistência ou retorno de algum sintoma relacionado com DRGE, sendo o baixo ganho ponderal o sintoma que mais persistiu.

Em relação aos seis pacientes com esofagite erosiva no exame de pós-operatório, um com encefalopatia crônica, evoluiu com melhora clínica total, quatro apresentaram melhora clínica parcial, sendo a metade encefalopatas crônicos e apenas um paciente, com ECNP, manteve sintomas após a cirurgia, aquele com subestenose de esôfago e esofagite grau D de Los Angeles.

Os dois pacientes com esôfago de Barrett referiam melhora total dos sintomas no período pós-operatório.

Não houve relato de aparecimento de qualquer novo sintoma após o procedimento cirúrgico. Na Tabela 3 há o resumo dos resultados gerais.

\section{DISCUSSÃO}

A endoscopia digestiva alta, realizada no período pósoperatório de fundoplicatura, identificou anormalidades da válvula antirrefluxo em cerca de $10 \%$ de 45 crianças e adolescentes. Esta avaliação foi realizada entre o primeiro e segundo ano após a fundoplicatura e incluiu $57,8 \%$ de pacientes encefalopatas crônicos. Desmanche total da válvula antirrefluxo foi identificado em um paciente. A endoscopia digestiva alta permitiu a detecção dos diagnósticos pós-operatórios de esofagite péptica, subestenose péptica de esôfago e suspeita de esôfago de Barrett, além de permitir a obtenção de biopsias, sob visualização direta, dos

\begin{tabular}{lcc}
\hline & $\mathrm{n}$ & $\%$ \\
\hline Diagnóstico ECNP & 19 & 42,2 \\
Não & 26 & 57,8 \\
Sim & & \\
EDA pré-op & 03 & 11,5 \\
Normal & 16 & 61,5 \\
Esofagite & 01 & 3,9 \\
(Sub)estenose & 06 & 23,1 \\
Esofagite + (sub)estenose & & \\
Status da fundoplicatura & 41 & 91,1 \\
Continente, bem posicionada & 03 & 6,7 \\
Intratorácica & 01 & 2,2 \\
Desmanche total/parcial & & \\
EDA pós-op & 36 & 80,0 \\
Normal & 05 & 11,2 \\
Esofagite & 01 & 2,2 \\
(Sub)estenose & 01 & 2,2 \\
Esofagite + (sub)estenose & 01 & 2,2 \\
Suspeita de Barrett & 01 & 2,2 \\
Subestenose+ suspeita Barrett & & \\
\hline
\end{tabular}

TABELA 3. Resumo dos resultados

locais macroscopicamente alterados. Recidiva ou persistência da DRGE foi identificada em seis pacientes.

Intercorrências relacionadas à cirurgia de fundoplicatura estão associadas a maior gravidade da DRGE e essa ocorrência é frequentemente relacionada a algumas características clínicas, entre elas associação com pneumopatia e encefalopatia crônicas ${ }^{(6,}$ ${ }^{12,14)}$. Considerando essa premissa, a casuística analisada no presente estudo incluiu mais da metade dos pacientes pertencentes a grupo de risco para DRGE grave, implicando em maior morbidade cirúrgica e maior risco para desmanche da válvula.

Apesar das descrições de maior frequência de complicações cirúrgicas no paciente encefalopata, este fato não foi estatisticamente comprovado, na presente casuística. $\mathrm{O}$ número total de complicações cirúrgicas imediatas ou precoces foi relativamente alto $(15,5 \%)$, mas similar ao observado por outros autores, que relataram percentuais variando de $0 \%$ a $15 \%$ em crianças e adolescentes operados, predominantemente, pela técnica de Nissen $^{(2,3,7,28,38)}$. As complicações ocorridas no pós-operatório foram estatisticamente mais frequentes entre os pacientes que realizaram a fundoplicatura de Nissen por laparotomia. Neste grupo estão incluídas as crianças abaixo de 1 ano, nas quais a laparoscopia não foi utilizada por limitações de equipamento. Além disso, o maior índice de complicações está claramente ligado à presença de complicações relacionadas à ferida operatória, as quais obviamente não ocorrem após a laparoscopia.

O percentual de anormalidades da válvula antirrefluxo no presente estudo foi de 8,9\% (4 em 45 pacientes), compatível com o que é descrito na literatura, com variação de $5 \%$ a $20 \%{ }^{(3,28,37,38)}$. Os resultados desta série diferiram do que foi encontrado no estudo de GUARINO et al. ${ }^{(1)}$, no qual se identificou anormalidades na válvula antirrefluxo em quase $50 \%$ das 109 crianças neurologicamente saudáveis e sem sintomas respiratórios, avaliadas com tempo médio de fundoplicatura de 18 meses. ZEID et al. ${ }^{(38)}$ encontraram anormalidades na válvula antirrefluxo em 3 de 26 crianças neurologicamente saudáveis, com média de seguimento de 28 meses. Os autores observaram que dois dos três pacientes com anormalidades na 
válvula antirrefluxo (desmanche total) apresentavam estenose de esôfago no pré-operatório, fato semelhante ao observado no presente estudo, no qual o único paciente com desmanche total da válvula antirrefluxo apresentou esofagite grau D de Los Angeles associada à subestenose péptica de esôfago. Aparentemente, casos com esofagite pré-operatória mais grave dificultam a confecção cirúrgica da válvula, estando provavelmente associados a maior índice de insucesso. No presente estudo não foi observado associação estatística entre esofagite grave pré-operatória e sucesso da fundoplicatura.

Anormalidades na válvula antirrefluxo associaram-se à esofagite péptica, de maneira semelhante à encontrada por GUARINO et al. ${ }^{(11)}$ nos estudos endoscópicos realizados entre 1 a 2 anos de pós-operatório. A fundoplicatura intacta parece prevenir a recurrência da DRGE e a recidiva da doença é mais frequente quando o exame endoscópico mostra defeitos na válvula antirrefluxo.

Segundo JAILWALA et al. ${ }^{(16)}$, a avaliação de deslocamento e desmanche parcial ou completo da válvula antirrefluxo tem maior precisão quando avaliada pelo exame endoscópico que pelo estudo radiológico contrastado. Esses autores encontraram desmanche da válvula antirrefluxo em três de quatro pacientes com esofagite péptica à endoscopia digestiva alta. $\mathrm{O}$ estudo radiológico contrastado pode detectar anormalidades estruturais em casos extremos, sendo a visualização direta oferecida pela EDA mais sensível.

Estudos envolvendo apenas encefalopatas crônicos e a avaliação no pós-operatório de fundoplicatura evidenciam retorno da DRGE, confirmado por exame complementar, em $40 \%$ a $48 \%$ dos casos, e necessidade de nova cirurgia em $15 \%$ a $25 \%$ dos $\operatorname{casos}^{(10,24)}$. GOESSLER et al. ${ }^{(10)}$ observaram que a recurrência do refluxo teve correlação significativa com o grau de desnutrição encontrada no pré-operatório, sendo mais prevalente nos desnutridos graves. Alguns autores acreditam que o retardo do esvaziamento gástrico também pode contribuir para maior insucesso cirúrgico ${ }^{(7,29)}$, assim como episódios de convulsão, espasticidade e doenças respiratórias associadas, situações em que ocorreria aumento da pressão intra-abdominal, favorecendo anormalidades na fundoplicatura e retorno do refluxo ${ }^{(19,20)}$.

Hérnia paraesofagiana foi diagnosticada em cinco pacientes $(11,1 \%)$, sendo quatro encefalopatas crônicos. Ocorrência semelhante à descrita na literatura, com frequência variando de $2,6 \%$ a $16,8 \%{ }^{(1,7,28)}$. Esta complicação pode levar à recurrência da DRGE por favorecer alterações na válvula antirrefluxo. A realização de hiatoplastia robusta é recomendada para evitar esta complicação ${ }^{(1,7,17,28)}$.

Em estudo multicêntrico recente, foi observado que a prevalência de esôfago de Barrett, com suspeição ao exame de EDA em crianças e adolescentes é baixa e reduz-se para menos da metade quando considerado o diagnóstico histológico confirmatório $^{(5)}$. JONES et al. ${ }^{(18)}$ relataram que pouco mais do que $20 \%$ dos casos de adultos com esôfago revestido por epitélio colunar podem ter o diagnóstico de metaplasia intestinal especializada em exames subsequentes, o que justificaria o seguimento destes pacientes. É possível que a presença da subestenose péptica de esôfago, identificada na endoscopia de pré-operatório, tenha dificultado a avaliação adequada da mucosa do esôfago distal, diminuindo a sensibilidade do exame para suspeita de esôfago de Barrett em um dos pacientes e, assim, prejudicado a coleta de biopsias para o diagnóstico. Como o aspecto endoscópico da válvula antirrefluxo estava preservado neste paciente, é pouco provável que o desenvolvimento de esôfago de Barrett tenha ocorrido após a cirurgia. O mais provável é que o esôfago de Barrett já existia no pré-operatório e não foi corretamente diagnosticado.

Em crianças, pouca correlação tem sido encontrada entre os achados histológicos de esofagite e sintomas, achados endoscópicos e achados de monitorização prolongada do $\mathrm{pH}$ intraesofágico, tanto qualitativa como quantitativamente ${ }^{(31,36)}$. Enquanto várias anormalidades são descritas na esofagite de refluxo, o critério histológico varia entre os mais experientes patologistas ${ }^{(13)}$ e os achados não são específicos ${ }^{(33)}$. No presente estudo optou-se pelo critério histológico apresentado por GEBOES et al. ${ }^{(9)}$, em 1991 , e também utilizado por VANDENPLAS et al. ${ }^{(34)}$. A esofagite de refluxo apresenta-se como lesão irregular, com lacunas, devendose considerar a possibilidade de um paciente com esofagite endoscópica erosiva apresentar exame histológico normal ou com alterações de leve a grave intensidade ${ }^{(13)}$.

Os achados desta série de associação entre anormalidades na válvula antirrefluxo e esofagite, indicam a necessidade de realizar biopsia esofágica quando há anormalidade na válvula antirrefluxo, mesmo frente a aspecto macroscopicamente normal da mucosa esofágica.

Os resultados relacionados aos sintomas no pós-operatório são compatíveis com os resultados encontrados em estudos anteriores, em adultos e em crianças e adolescentes, mostrando baixa correlação entre sintomatologia e achados endoscópicos $(4,8,11,24,32)$. Os sintomas de DRGE podem persistir ou recorrer mesmo sem anormalidades na válvula antirrefluxo, e pacientes com anormalidades relacionadas ao esôfago, como esofagite erosiva ou esôfago de Barrett, podem ser assintomáticos. Estes resultados são recurrentes em estudos que incluíram pacientes com encefalopatia crônica e neurologicamente saudáveis ${ }^{(11,24,32)}$. $\mathrm{Na}$ casuística estudada, a maior parte dos pacientes com anormalidades relacionadas ao esôfago eram encefalopatas crônicos, o que prejudicou a confiabilidade quanto à presença ou não de sintomas no período pós-operatório.

A EDA foi útil para avaliar os pacientes em pós-operatório tardio de fundoplicatura para DRGE, além de informar objetivamente sobre a condição da válvula antirrefluxo, permitiu diagnosticar a presença de subestenose, esofagite péptica e casos suspeitos de esôfago de Barrett, com recurso de realizar biopsias para confirmação deste último diagnóstico. Acreditase que seja método complementar necessário para avaliação de pacientes que realizaram fundoplicatura, uma vez que o achado de esofagite persistente ou recurrente apresenta baixa correlação com presença de sintomas clínicos e é específico para falha do tratamento cirúrgico.

São desejáveis outros estudos com maiores casuísticas, desenhos prospectivos e longitudinais, com o objetivo de definir um programa de seguimento para os pacientes operados por DRGE. 
Vicente AMB, Cardoso SR, Servidoni MFCP, Meirelles LR, Silva JMB, Costa-Pinto EAL. Clinical and endoscopic outcome after Nissen fundoplication for gastroesophageal reflux disease. Arq Gastroenterol. 2009;46(1):138-43.

ABSTRACT - Context - The surgical treatment of gastroesophageal reflux disease is indicated in patients with a chronic condition when proton pump inhibitors therapy is ineffective or when symptoms are persistently recurrent. Fundoplication failure occurrence has been detected in some patients and highlights a needing of monitoring esophageal post-surgical condition since that clinical symptoms are not sensitive enough for indicating fundoplication failure. Aims - To identify the frequency of a disrupted wrap in the postoperative period and to evaluate esophageal complications related with gastroesophageal reflux recurrence in children and adolescents. Methods - The study was cross-sectional, prospective and descriptive, including 45 patients (16 months-16.9 years) who had undergone Nissen fundoplication in a school hospital. Twenty six patients (57.8\%) were neurologically impaired. Upper gastrointestinal endoscopy was performed in order to determine fundoplication integrity, endoscopic and histopathological esophageal condition. Upper gastrointestinal endoscopies were performed from 12 up to 30 months after surgery. Results - Patent wrap was identified in 41 patients $(91.1 \%)$. Recurrent peptic esophagitis was found in 6 of 45 patients, 2 of which required a second fundoplication and other two had Barrett esophagus. Endoscopic peptic esophagitis was associated with a defective wrap $(P=0.005)$. Conclusions - Fundoplication was effective for treating esophagitis, even in patients with previous esophageal stenosis. Endoscopic follow up may detect surgery failure in children undergone anti-reflux surgery even in asymptomatic patients.

HEADINGS - Fundoplication. Gastroesophageal reflux, surgery. Esophagitis. Esophageal stenosis. Barrett esophagus. Endoscopy, gastrointestinal. Child

\section{REFERÊNCIAS}

1. Alrabeeah A, Giacomantonio M, Gillis DA. Paraesophageal hernia after Nissen fundoplication: a real complication in pediatric patients [abstract]. J Pediatr Surg. 1988;23:766-8.

2. Davidson BR, Hurd DM, Johnstone MS. Nissen fundoplication and pyloroplasty in the management of gastro-oesophageal reflux in children. Br J Surg. 1987;74:488-90.

3. Dedinsky GK, Vane DW, Black T, Turner MK, West KW, Grosfeld JL. Complications and reoperation after Nissen fundoplication in childhood. Am J Surg. 1987;153:177-82.

4. Draaisma WA, Rijnhart-de Jong HG, Broeders IA, Smout AJ, Furnee EJ, Gooszen HG. Five-year subjective and objective results of laparoscopic and conventional Nissen fundoplication: a randomized trial. Ann Surg. 2006;244:34-41.

5. El-Serag HB, Gilger MA, Shub MD, Richardson P, Bancroft J. The prevalence of suspect Barrett's esophagus in children and adolescents: a multicenter endoscopic study. Gastrointest Endosc. 2006;64:671-5.

6. Ferreira CT, Carvalho E. Refluxo gastroesofágico. In: Ferreira CT, Carvalho E, Silva L., editores. Gastroenterologia e hepatologia em pediatria: diagnóstico e tratamento. Rio de Janeiro: Medsi; 2003. p.3-29.

7. Fonkalsrud EW, Foglia RP, Ament ME, Berquist W, Vargas J. Operative treatment for the gastroesophageal reflux syndrome in children. J Pediatr Surg. 1989;24:525-9.

8. Galvani C, Fisichella PM, Gorodner MV, Perretta S, Patti MG. Symptoms are a poor indicator of reflux status after fundoplication for gastroesophageal reflux disease. Arch Surg. 2003;138:514-9.

9. Geboes K, Ectors N, Vantrappen G. Inflammatory disease of the oesophagus. Hepatogastroenterology. 1991;38(suppl 1):s26-s30.

10. Goessler A, Zeyringer AH, Hoellwarth. Recurrent gastroesophageal reflux in neurologically impaired patients after fundoplication. Acta Pediatr. 2007;96:87-93.

11. Guarino N, Ceriati E, Zaccara A, La Sala E, De Peppo F, Dall'Oglio L. Is endoscopic follow-up needed in pediatric patients who undergo surgery for GERD? Gastrointest Endosc. 2002;55:387-9.

12. Hassal E. Decisions in diagnosing and managing chronic gastroesophageal reflux disease in children. J Pediatr. 2005;146(suppl 1):s3-s12.

13. Hassal E. Esophageal biopsy in children-essential, valuable, or a waste of time? it all depends. J Pediatr Gastroenterol Nutr. 2005;41:s24-s7.

14. Hassal E. Outcomes of fundoplication: causes for concern, newer options. Arch Dis Child. 2005;90:1047-52.

15. Hillemeier AC. Gastroesophageal reflux. In: Walker WA, Duric PR, Hamilton JR, Walker-Smith JA, Watkins JB, editors. Pediatric gastrointestinal disease: pathophysiology, diagnosis, management. 3rd ed. Hamilton, Ontario: B.C. Decker; 2000.p.289-97.

16. Jailwala J, Massey B, Staff D, Shaker R, Hogan W. Post-fundoplication symptoms: the role for endoscopic assessment of fundoplication integrity. Gastrointest Endosc. 2001;54:351-6

17. Johnson DA, Younes Z, Hogan WJ. Endoscopic assessment of hiatal hernia repair. Gastrointest Endosc. 2000;52:650-9.

18. Jones T, Sharma P, Daaboul B, Cherian R, Mayo M, Topalovski M, Weston AP. Yield of intestinal metaplasia in patients with suspected short-segment Barrett's esophagus (SSBE) on repeat endoscopy. Dig Dis Sci. 2002;47:2108-11.

19. Kawahara H, Okuyama H, Kubota A, OUE T, Tazuke Y, Yagi M. Can laparoscopic antireflux surgery improve the quality of life in children with neurologic and neuromuscular handicaps? J Pediatr Surg. 2004;39:1761-4.

20. Kimber C, Kiely EM, Spitz L. The failure rate of surgery for gastro-oesophageal reflux. J Pediatr Surg. 1998;33:64-6.

21. Kusano M, Ino K, Yamada T, Kawamura O, Toki M, Ohwada T, Kikuchi K, Shirota T, Kimura M, Miyazaki M, Nakamura K, Igarashi S, Tomizawa M, Tamura T, Sekiguchi T,
Mori M. Interobserver and intraobserver variation in endoscopic assessment of GERD using the "Los Angeles" classification. Gastrointest Endosc. 1999;49:700-4.

22. Lundell LR, Dent J, Bennett JR, Blum AL, Armstrong D, Galmiche JP, Johnson F, Hongo M, Richter JE, Spechler SJ, Tytgat GN, Wallin L. Endoscopic assessment of oesophagitis: clinical and functional correlates and further validation of the Los Angeles classification. Gut. 1999;45:172-80

23. Luostarinen M. Nissen fundoplication for reflux esophagitis. Long-term clinical and endoscopic results in 109 of 127 patients. Ann Surg. 1993;217:329-37.

24. Martinez DA, Ginn-Pease ME, Caniano DA. Recognition of recurrent gastroesophageal reflux following antireflux surgery in the neurologically disabled child: high index of suspicion and definitive evaluation. J Pediatr Surg. 1992;27:983-90.

25. O'Hanrahan T, Marples M, Bancewicz J. Recurrent reflux and wrap disruption after Nissen fundoplication: detection, incidence and timing. Br J Surg. 1990;77:545-7.

26. Orenstein SR. Gastroesophageal reflux. In: Wyllie R, Hyams J., editors. Pediatric gastrointestinal disease. 2nd ed. Philadelphia: Saunders; 1999. p.164-87.

27. Pandolfino JE, Vakil NB, Kahrilas PJ. Comparison of inter and intraobserver consistency for grading of oesophagitis by expert and trainee endoscopists. Gastrointest Endosc. 2002;56:639-43.

28. Parikh D, Tam PKH. Results of fundoplication in a UK paediatric centre. Br J Surg. 1991;78:346-8.

29. Ramachandran V,Ashcraft KW, Sharp RJ, Murphy PJ, Snyder CL, Gittes GK, Bickler SW Thal fundoplication in neurologically impaired children. Pediatr Surg. 1996;31:819-22.

30. Rath HC, Timmer A, Kunkel C, Endlicher E, Grossmann J, Hellerbrand C. Comparison of interobserver agreement for different scoring systems for reflux oesophagitis: impact of level of experience. Gastrointest Endosc. 2004;60:44-9.

31. Salvatore S, Hauser B, Vandemele K, Novario R, Vandenplas Y. Gastroesophageal reflux disease in infants: how much is predictable with questionnaires, $\mathrm{pH}$-metry, endoscopy and histology? J Pediatr Gastroenterol Nutr. 2005;40:210-5.

32. Strecker-Mc Graw MK, Lorenz ML, Hendrickson M, Jolley SG, Tunell WP. Persistent gastroeophageal reflux disease after antireflux surgery in children: I. immediate postoperative evaluation using extended esophageal $\mathrm{pH}$ monitoring. J Pediatr Surg. 1998;33:1623-7.

33. Takubo K, Honma N, Aryal G, Swabe M, Arai T, Tanaka Y, Mafune K, Iwakiri K. Is there a set of histologic changes that are invariably reflux associated? Arch Pathol Lab Med. 2005;129:159-63.

34. Vandenplas Y, Ashkenazi A, Belli D, Boige N, Bouquet J, Cadranel S, Cezard JP, Cucchiara S, Dupont C, Geboes K, et al. A proposition for the diagnosis and treatment of gastro-oesophageal reflux disease in children: a report from a working group on gastro-oesophageal reflux disease. Working Group of the European Society of Pediatric Gastro-enterology and Nutrition (ESPGAN). Eur J Pediatr. 1993;152:704-11.

35. Vandenplas Y. Gastroesophageal reflux: medical treatment. J Pediatr Gastroenterol Nutr. 2005;41(suppl 1):s41-s2.

36. Vieira MC, Pisani JC, Mulinari RA. Diagnóstico de esofagite de refluxo em lactentes: a histologia do esôfago distal deve complementar a endoscopia digestiva alta. J Pediatr (Rio J). 2004;80:197-202

37. Wheatley MJ, Coran AG, Wesley JR, Oldham KT, Turnage RH. Redo fundoplication in infants and children with recurrent gastroesophageal reflux. J Pediatr Surg. 1991;26: 758-61.

38. Zeid MA, Kandel T, el-Shobary M, Talaat AA, Fouad A, el-Enien AA, el-Badrawy T, el-Hak NG, el-Wahab MA, Ezzat F. Nissen fundoplication in infants and children: long-term clinical study. Hepatogastroenterology. 2004;51:697-700.

Recebido em 10/4/2008 Aprovado em 7/8/2008 